\title{
A atividade física para gestantes: análise da formação específica
}

\author{
Physical activity for pregnant women: analysis of specific formation \\ La actividad física para gestantes: análisis de la formación específica
}

\author{
Alexandre Scherer ${ }^{\mathrm{I}}$, Marcella Souza Massa Pires De Oliveira ${ }^{\mathrm{II}}$, Fernanda Mariana Scherer ${ }^{\mathrm{III}}$
}

\begin{abstract}
Resumo
Este estudo de cunho qualitativo objetivou compreender que tipo de formação tem os profissionais de Educação Física que trabalham com gestantes. Inicialmente, refletiu-se sobre a gestação, suas características, a influência dos exercícios físicos e a formação profissional. Foi utilizada a entrevista semiestruturada e a análise de currículos em Educação Física. As informações foram categorizadas. As conclusões apontam que os conhecimentos relevantes para a intervenção com grávidas estão ligados aos aspectos biológicos e de treinamento físico e se efetivam mais expressivamente através de cursos de extensão e pela experiência no acompanhamento do processo de gestação.
\end{abstract}

Palavras-chave: Educação Física e Treinamento; Capacitação Profissional; Gestantes

\begin{abstract}
This qualitative study aimed at understanding what kind of training has Physical Education professionals who work with pregnant women. Initially, it was reflected on pregnancy, its characteristics, the influence of physical exercises and the professional training. Semi-structured interviews and curriculum analysis in Physical Education were used. The information was categorized. The conclusions point out that the relevant knowledge for the intervention with pregnant women is linked to biological aspects and physical training and is carried out more expressively through extension courses and experience in monitoring the pregnancy process.
\end{abstract}

Keywords: Physical Education and Training; Professional Training; Pregnant Women

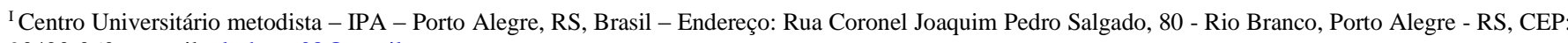
90420-060 - e-mail: alscherer08@gmail.com

${ }^{\text {II }}$ Centro Universitário metodista - IPA - e-mail: m.personal@live.com

${ }^{\text {III } C e n t r o ~ U n i v e r s i t a ́ r i o ~ m e t o d i s t a ~-~ I P A ~-~ e-m a i l: ~ f e r n a n d a . s c h e r e r 97 @ g m a i l . c o m ~}$ 


\section{Resumen}

El presente estudio cualitativo objetivó comprender qué tipo de formación tienen los profesionales de Educación Física que trabajan con gestantes. Al principio, se reflexionó sobre la gestación, sus características, la influencia de los ejercicios físicos y la formación profesional. Se utilizó la entrevista semiestructurada y el análisis de las malas curriculares de formación en Educación Física. Las informaciones han sido categorizadas. Las conclusiones apuntan que los conocimientos relevantes para la intervención con gestantes están vinculado a aspectos biológicos y entrenamiento físico y se realiza de manera más significativa a través de cursos de extensión y por la vivencia en el seguimiento del proceso de gestación.

Palabras clave: Educación y Entrenamiento Físico; Capacitación Profesional; Gestantes

\section{Introdução}

Diante do contexto sociocultural que se apresenta nos dias de hoje, em que a saúde, o bem estar e a estética caminham cada vez mais próximos e se misturam dentro das academias, é possível perceber mais um grupo ganhando um espaço considerável neste ambiente: as gestantes. Essa é uma lacuna ainda em aberto para muitos profissionais da Educação Física no que diz respeito ao conhecimento e experiência, como aspectos nutricionais, condições físicas e funcionais da gestante durante a atividade física, trabalho de parto e prematuridade e atividade física e crescimento fetal (GALLUP, 1999; BATISTA et al., 2003).

Para superar esse desafio supôs-se que a formação em Educação Física pudesse colaborar com a inserção de conhecimentos e experiências sobre o tema. Esse é o foco deste estudo que se centra em dialogar com profissionais da área que trabalham com gestantes, considerando que há uma necessidade de intervenção diferenciada. A questão que norteou o estudo foi: que conhecimentos e experiências são necessários aos profissionais de Educação Física para atuar com mulheres no período gestacional?

Para tanto, os objetivos desta pesquisa se centraram em compreender que tipo de formação tem os profissionais de Educação Física que trabalham com atividade física para gestantes, conhecer as particularidades do trabalho realizado com gestantes e identificar os conhecimentos e as experiências mais relevantes aos profissionais de Educação Física para atuar com gestantes.

Para isso, estudaram-se as características bioquímicas e fisiológicas das gestantes, bem como se analisaram as referências que tratam de exercícios físicos para essa comunidade específica. Em seguida, se contextualizou sobre a formação em Educação Física: do geral ao específico estabelecendo relações entre os conhecimentos desenvolvidos e que podem ser voltados às gestantes. A metodologia qualitativa foi utilizada e envolveu uma entrevista semiestruturada com seis profissionais que intervém com a 
população pesquisada. Após a realização das entrevistas, os dados foram interpretados e posteriormente categorizados em três categorias.

\section{A gestação: modificações bioquímicas e fisiológicas}

As adaptações no organismo materno vão muito além do que nossos olhos podem enxergar e do que as próprias gestantes podem sentir. Segundo Prates, Santos e Moretto (2005), essas adaptações são atribuídas aos hormônios e à mecânica do crescimento do útero e demais órgãos envolvidos etais modificações protegem as funções fisiológicas normais da mulher, atendem as demandas metabólicas do período gestacional e suprem as necessidades fetais.

No sistema reprodutivo, por exemplo, Stephenson e O’Connor (2004) afirmam que a maior parte das modificações de suas estruturas ocorre devido à ação dos hormônios estrógeno e progesterona. Tais hormônios acabam por influenciar no relaxamento dos ligamentos pélvicos e do tecido conjuntivo em geral.

Para Valadares (2002), no sistema cardiovascular e hematológico, o volume sanguíneo aumenta em resposta à demanda nutricional e de oxigênio do feto e ainda compensa a perda de sangue ocorrida durante o parto. Esse aumento se dá em diferentes proporções de plasma e hemoglobina, o que pode acarretar a anemia fisiológica que, como a anemia adquirida, ocorre devido à falta ou má absorção de ferro, mas podem ser atenuadas com a suplementação de ferro e a ingesta de ácido ascórbico. Para a autora, em relação ao sistema respiratório, a gestação pode levar muitas mulheres a sentir falta de ar ou dispneia, muitas vezes causada devido aos níveis aumentados de progesterona. Evans (2010) complementa que ao final da gestação a dispneia acomete as mulheres quando o diafragma é pressionado e deslocado para cima devido ao crescimento do feto.

Em relação ao sistema renal esse mesmo autor afirma que há um aumento da frequência urinária e isso acontece em decorrência de alterações fisiológicas, como o aumento da pressão vesical pelo crescimento do útero. Almeida, Candido e Oliveira Netto (2020) afirmam que também existe maior possibilidade de haver sintomas de incontinência urinária que podem ser causados devido a diminuição da função muscular do assoalho pélvico.

A gestação também afeta o sistema musculoesquelético e sobre esse aspecto,Valadares (2002) explica que devido ao centro de gravidade deslocado a frente, há uma projeção do corpo para trás (hiperlordose), um distanciamento dos pés, além da projeção à frente da coluna cervical, o que pode resultar em uma lombalgia ou uma cervicalgia. 
Quanto ao sistema endócrino a autora refere-se à ação dos principais hormônios: progesterona e estrógeno; a progesterona é produzida pelo corpo lúteo até cerca de 10 semanas de gestação e gera a redução da tonicidade da musculatura lisa em órgãos maternos; o estrógeno tem sua liberação no corpo causando uma retenção de líquidos gerando um aumento na flexibilidade das articulações pélvicas e atuando juntamente com a prolactina nos ductos mamários; e a relaxina que é o hormônio produzido pelo corpo lúteo gravídico e está associado à flexibilidade e extensibilidade das articulações.

Stephenson e O'Connor (2004) contribuem ainda para entender a atuação de outros hormônios como o hCG (gonadotropina coriônica humana) que é produzido pela placenta e pelo embrião fecundado e tem a função de aumentar e manter o leito endometrial e impedir a menstruação, além de ser responsável pela maior secreção de estrógeno e progesterona através do corpo lúteo e o hormônio hPL (lactogênio placentário humano) que atua como antagonista da insulina, liberando ácidos graxos para fornecer energia ao metabolismo materno e fetal.

Fica claro que a mulher é acometida desde a concepção até o parto por uma série de modificações fisiológicas que a deixam em estado diferenciado de uma situação normal, entretanto, observa-se que a maiores influências são bioquímicas nas quais os autores citados acima consideram que a gestante passa por uma "revolução hormonal".

\section{A gestação e o exercício físico}

Souza (2002) afirma que é imprescindível que a mulher adote maiores cuidados com relação à postura corporal. A execução de exercícios durante a gravidez evita o ganho excessivo de peso, reduz o estresse cardiovascular, estimula a boa postura, previne dores nas regiões da coluna vertebral, melhora a imagem corporal e, consequentemente, o estado psicológico, além de facilitar o tempo do desprendimento fetal em partos vaginais. $\mathrm{O}$ autor afirma ainda que em caso de baixa estima, recomendam-se atividades feitas em grupo, trabalhando a conscientização postural, a reeducação da atividade respiratória e os relaxamentos musculares através de exercícios e massagens.

Ramos (2002) relata que o primeiro e o terceiro trimestre de gravidez merecem atenção especial e cita fatores que interferem a elaboração do treino, como por exemplo o grau de condicionamento físico, a fase da gestação, a motivação pessoal, a limitação médica e o tempo disponível para o cuidado de si.

Em resposta ao exercício, Stephenson e O’Connor (2004) relatam que as alterações de frequência cardíaca fetal podem indicar asfixia, porém esse diagnóstico só acontece se houver redução de 50\% do 
fluxo sanguíneo uterino. Os autores afirmam ainda que o volume de oxigênio uterino é mantido mesmo ocorrendo a redução acentuada do fluxo sanguíneo uterino durante o exercício, sendo que tanto as demandas maternas de oxigênio quanto as uterinas tornam-se aumentadas devido à expansão do feto e da placenta. Eles consideram ainda que a pressão arterial da gestante pode aumentar até $20 \%$ em resposta ao exercício físico. Além dessas alterações, o exercício físico traz benefícios para a gestante e para o feto. Segundo as 2019 Canadian Guideline for Physical Activity Throughout Pregnancy, realizar, pelo menos 150 minutos semanais de atividades com intensidade moderada, aumenta os benefícios durante a gestação e diminui o risco de complicações que podem ocorrer nesse período.

Souza (2002) afirma, porém, que cada gestante deve ser tratada de forma única, respeitando sua individualidade. Para o autor, são exercícios que devem ser evitados de forma geral aqueles que aumentem a pressão intra-abdominal. Sugere que se utilizem diferentes posições e tomem-se as medidas necessárias a um programa de exercícios eficaz para a gravidez.

Os estudos realizados até aqui mostram que as gestantes têm características próprias. Existem alterações fisiológicas, bioquímicas e psicológicas específicas. Nesse sentido, é necessário saber se a formação em Educação Física colabora ou não com esse processo e tais considerações serão abordadas no próximo tópico.

\section{A formação profissional em educação física: do geral ao específico}

O debate da formação em Educação Física para o atendimento às gestantes é bastante complexo por se tratar de uma área epistemológica que abrange educação e saúde(ZAVORSKY; LONGO, 2011).Atualmente o processo de formação em Educação Física sofre algumas modificações devido a debates no que tange a capacitação de profissionais aptos a trabalhar junto a uma sociedade em constante mudança a fim de suprir as suas necessidades, não somente no ambiente escolar, como nos diversos espaços ligados ao movimento humano(SCHERER, 2017).

As diretrizes curriculares atuais se distinguem do modelo anterior proporcionando ao licenciado e ao bacharel os cabidos saberes. Apesar do debate epistemológico e jurídico do tema no Brasil, sugere-se pensar que ao licenciado propõem-se prioritariamente as práticas pedagógicas inerentes às diversas disciplinas que dispõe o curso, permitindo-lhe atuação em campo específico (escola). Ao bacharel cabem as práticas desenvolvidas em ambiente não escolar, um conhecimento que não é voltado necessariamente à educação, mas sim para obtenção de resultados e melhoria de performance, independente da sua abrangência (saúde, bem-estar, estética, rendimento, entre outras). 
A partir das posições discutidas acima, este estudo centrou-se na formação do bacharel em Educação Física e as relações com os conhecimentos e competências para intervenção com gestantes. Menestrina (2005) explana que a sociedade necessita de profissionais que atendam às exigências da contínua mudança ocorrida no estilo de vida do ser humano, atenta para a obtenção de saúde através da qualidade de vida e bem-estar atingidos através da atividade física.

Dentre os conhecimentos relativos à saúde individual e coletiva destacam-se o lazer, as formas de expressão e as vivências corporais cotidianas. É necessário ao profissional da Educação Física a ciência das diversas formas de proporcionar bem-estar, bem como os meios de expressão não verbais dos sentimentos e emoções e ainda ter noção do repertório de movimentos executados diariamente pelos indivíduos e a finalidade dos mesmos, para então garantir que haja o aprimoramento e a otimização de tais movimentos em prol da saúde.

Para Menestrina (2005), o profissional de Educação Física que atua na promoção da saúde, deve possuir uma formação adequada, tendo conhecimento biológico, no que tange a individualidade do ser; conhecimento psicopedagógico que implica a maneira pela qual é desenvolvida a ação, o planejamento e avaliação dos resultados; conhecimento sociocultural que dá dimensão às atividades e expressões cultivadas pela sociedade; e o conhecimento técnico que se subdivide nas diferentes possibilidades de atuação profissional. Para isso, são necessários saberes teóricos e práticos que possibilitem a execução de procedimentos adequados à natureza dos praticantes.

As disciplinas analisadas nos currículos de Bacharelado em Educação Física e que tangem a área da saúde citados anteriormente estão: a pedagogia aplicada a educação, a anatomia humana, o desenvolvimento humano, a fisiologia (básica e do exercício), a sociologia e a psicologia aplicada à Educação Física, a Educação Física adaptada, os primeiros socorros, princípios e diretrizes do SUS, além das disciplinas especificas do exercício esportivo e demais atividades (lazer e recreação, atividade para a terceira idade, entre outras).

Em relação ao exercício físico aplicado a gestantes, é possível que haja vínculos entre as disciplinas de fisiologia, de desenvolvimento humano, ao longo das disciplinas esportivas, na educação física adaptada, no desporto adaptado, no treinamento físico em populações especiais, na atividade motora adaptada ou na atividade para grupos especiais.

Encontraram-se também atividades de formação continuada voltadas à saúde da mulher, como o curso de especialização voltado à atenção integral à saúde da mulher, como o de especialização atividade física adaptada e saúde, além de diversos cursos de extensão, inclusive voltados à prescrição de exercícios físicos para gestantes. 
Apesar de encontrarmos vestígios de que o tema "exercício físico e gestação" aparece na formação inicial e continuada, ela parece ser incipiente, entretanto o mercado é crescente e promissor tanto no que diz respeito à atuação diretamente com o público, quanto às pesquisas relacionadas.

\section{Caracterização da pesquisa}

A presente pesquisa é caracterizada pelo método qualitativo devido a sua eficiência como técnica de abordagem do referido tema. Segundo Mynaio (2010), este método tem fundamento teórico e permite esclarecer processos sociais ainda pouco discutidos referentes a grupos particulares, propiciando a construção de novas abordagens, revisando e criando novos conceitos e categorias durante as investigações.

A investigação, que tratou de trabalho de conclusão de curso, foi realizada com 6 profissionais de Educação Física graduados em bacharelado ou licenciatura plena generalista, que atuavam com gestantes no ano de 2012 e que possuíam experiência na prescrição de exercícios específicos para essa população há pelo menos um ano. Alguns referenciais teóricos foram ampliados para a atualização deste artigo.

A escolha dos participantes foi realizada por conveniência, que segundo Creswell (2010) se trata de uma amostragem de não probabilidade, em que os respondentes são escolhidos de acordo com sua conveniência e disponibilidade, são grupos naturalmente formados ou voluntários.

Como instrumento de coleta de informações, foram utilizadas entrevistas semiestruturadas. Esse instrumento foi escolhido pelo fato de possibilitar um roteiro flexível de diálogo, permitindo que os entrevistados atribuam novas questões e aspectos relevantes à pesquisa. Além disso, foram analisadas as grades curriculares de sete cursos de bacharelado em Educação Física situadas no estado do Rio Grande do Sul no sentido de identificar disciplinas que pudessem discutir o exercício físico para gestantes.

A análise dos dados foi feita através do método de categorização que classifica as ideias dos sujeitos e relaciona-as com o referencial teórico. A categorização é um mecanismo fundamental para a razão e a comunicação humana, estabelecendo bases para muitos dos mais importantes processos mentais, tais como a percepção, a representação, a linguagem, a lógica e a aprendizagem (BARDIN, 2004). Para preservar a identidade dos participantes, eles foram identificados como entrevistados A, B, C, D, E e F. 


\section{Análise das informações}

A partir das discussões das informações trazidas através do referencial teórico e das evidências relatadas pelos participantes do estudo se chegou a três categorias que envolvem a formação para a intervenção com atividade física em gestantes: Conhecimentos básicos e específicos: definições e origem; O conhecimento através das experiências pessoais; A sensibilidade na pedagogia do ensino.

\section{Conhecimentos básicos e específicos: definições e origem}

De acordo com as entrevistas realizadas e com a análise das matrizes curriculares dos cursos de bacharelado em Educação Física, os conhecimentos básicos relacionados à saúde aparecem com grande relevância, principalmente a anatomia, fisiologia humana e do exercício, bioquímica e o treinamento físico.

Nesse sentido, os sujeitos manifestaram a necessidade de saber mais sobre o controle da intensidade do exercício levando em consideração a sua percepção individual das gestantes. Durante a atividade física o profissional pode instruir a mulher de forma a alcançar uma das classificações da escala de Borg, por exemplo, independente do ritmo, da força ou do número de repetições de cada exercício. Dessa forma, também pode ser utilizado o frequencímetro, desde que o profissional saiba os limites cardíacos máximos e utilize uma escala para consulta de valores que variam entre 50 a $80 \%$ desta frequência proporcional a idade.

Para a entrevistada "A":

O profissional tem que entender bastante de treinamento e de resposta ao exercício [...] o segredo do treinamento para gestantes está em tu conseguir ter um bom equilíbrio entre volume e intensidade, tempo de duração do exercício e resposta térmica. (Entrevista realizada em 10 de agosto de 2012).

A fala da entrevistada "A" referenda também a preocupação do cuidado com a hipertermia, que segundo Ramos (2002) e Stephenson e O'Connor (2004) pode levar à desidratação causando má formação do feto e precipitação do parto.

Quanto aos conhecimentos relacionados aos tipos de exercício físico, foram citados aqueles que cabem às modalidades de: hidroginástica, treino de ciclismo indoor, treinamento funcional, treinamento resistido com pesos e pilates, sempre complementados pelas adaptações necessárias ao público alvo. 
Se a mulher tem um histórico de vida ativa fisicamente, cessar suas atividades de maneira abrupta a partir da gravidez também poderá levar a transtornos. Contudo, não está proibido iniciar atividades físicas regulares durante o período gestacional. Todavia diante de qualquer situação de risco é imprescindível que o exercício seja paralisado imediatamente e permaneça suspenso até a avaliação médica.

Concorda-se com Evans (2010) ao afirmar que, em geral, a gestante não tem que limitar os exercícios físicos. O autor explica que, no entanto, deve-se evitar a fadiga mantendo a intensidade do exercício. Já as mulheres sedentárias antes da gestação não devem realizar exercícios aeróbios mais extenuantes que a caminhada.

Ramos (2002) relata que o primeiro e o terceiro trimestre merecem atenção especial e cita fatores que interferem a elaboração do treino, como por exemplo: o grau de condicionamento, a fase da gestação, a motivação, a limitação médica e o tempo disponível.

O quesito unânime entre os entrevistados, considerado o mais importante é aquele referente às alterações gravídicas. Como já citado no referencial teórico, as adaptações no organismo materno vão muito além do que nossos olhos podem enxergar e do que as próprias gestantes podem sentir. Segundo Prates, Santos e Moretto (2005), essas adaptações são atribuídas aos hormônios e à mecânica do crescimento do útero e demais órgãos envolvidos. Tais modificações protegem as funções fisiológicas normais da mulher, atendem as demandas metabólicas do período gestacional e suprem as necessidades fetais. Embora a mulher no período da gestação passe por uma revolução hormonal, nenhum dos entrevistados mencionou conhecimentos sobre bioquímica.

Alguns conhecimentos dos profissionais são adquiridos através da graduação, porém de forma muito superficial. Para a entrevistada "F":

Durante toda a graduação existiram aspectos relevantes quanto a conhecimentos gerais, porém falta especificidade para determinados grupos, ao menos para os grupos especiais mais comuns dentro do ambiente de treino, seja musculação ou aquáticos. (Entrevista realizada em 30 de agosto de 2012).

Diferentes visões aparecem na fala dos participantes do estudo sobre os conhecimentos específicos para trabalhar com gestantes. A entrevistada "E" acredita que foram relevantes os conhecimentos básicos que envolveram o treinamento físico, porém o envolvimento com pesquisa na universidade teve maior impacto. No caso do entrevistado "B", os conhecimentos básicos como a fisiologia humana, a fisiologia do exercício e a anatomia trabalhados na graduação foram os mais relevantes e utilizados com este público específico. 
A entrevistada "F" afirmou que a experiência realizada direto na empresa que atuava com gestantes aliada à participação em um curso de especialização na faculdade de medicina, na área de ginecologia-obstetrícia a ajudou a ter mais segurança em sua intervenção.

A entrevistada "E", assim como a entrevistada "D" também tiveram treinamento no local de trabalho atual. Tal treinamento abrange de forma teórica os aspectos fisiológicos e as respostas ao exercício físico durante a gestação e ainda tem a parte prática que inicia de forma gradual a partir da aferição de pressão arterial e da frequência cardíaca, até que se chegue à conquista de uma turma de gestantes.

Segundo a entrevistada "E":

Não há treinamento melhor do que a prática[...] poder trabalhar ao lado de especialistas antes de se aventurar sozinha com mulheres nesse momento tão especial, faz toda a diferença na hora de analisar o feedback que elas nos passam. (Entrevista realizada em 22 de agosto de 2012).

Cinco dos seis participantes afirmam ter feito cursos de extensão sobre exercício físico na gestação e então tomaram ciência das alterações gravídicas. Nota-se, então, a necessidade da busca por conhecimento teórico e prático que vai além da graduação para atuar com as gestantes.

\section{0 conhecimento através das experiências pessoais}

Nesta categoria deixa-se de falar sobre a formação acadêmica e centra-se nas experiências pessoais e o que elas acarretam na carreira profissional dos sujeitos pesquisados.

Todos os sujeitos tiveram contato de alguma forma com a gestação e com o nascimento de uma criança. Três profissionais já eram mães e o único participante do sexo masculino já era pai e acompanhou todo o processo, da gestação até o parto. As outras participantes acompanharam a gestação de pessoas próximas ou utilizaram de vídeos via internet, a fim de apropriarem-se de como esse fato ocorre.

A entrevistada "A" relata abaixo o seu processo pessoal: 
Ser mãe, passar pela gestação, com certeza modifica muito o trabalho. A vivência de cada processo, a vivência do parto, são coisas que enriquecem o conhecimento, por que, por exemplo, o trabalho de parto é um assunto que com certeza vai surgir durante as aulas e existem diversos exercícios que auxiliam na hora do parto, seja devido ao autoconhecimento que proporcionam, de saber contrair e relaxar o períneo ou por auxiliarem também na recuperação pós-parto e é bem complicado falar sobre parto sem entender o processo. (Entrevista realizada em10 de agosto de 2012).

Para a entrevistada "E":

Não sou mãe, mas eu tenho um afilhado que tem 6 meses, então, acabou que a mãe engravidou dele nesse período de um ano e meio que eu comecei a dar aula para gestantes e é uma amigona minha, então no dia que ela ficou sabendo que estava grávida ela me convidou para ser madrinha e eu acompanhei toda a gestação dela. Isso me ajudou para eu ter uma noção de como acontecem realmente as alterações, principalmente em relação às mudanças posturais, que por mais que a gente aprenda na teoria dificilmente temos oportunidade para analisar a postura das gestantes e eu tive a oportunidade de acompanhar direitinho o todo esse processo, até por que já a conheço há anos, enfim [...]. (Entrevista realizada em 22 de agosto de 2012).

Além disso, essa participante ressalta a importância das observações das alterações posturais quando existe a convivência com gestantes.

A entrevistada "F" não tem filhos e afirma que sentia a necessidade de acompanhar um parto pessoalmente e que antes disso buscava vídeos na internet. Tempo depois a participante teve a oportunidade de acompanhar pessoalmente essa ação, mas acredita que agregará ainda mais conhecimentos quando passar pelo processo, pois assistir via internet ou pessoalmente não tem grandes diferenças.

Já a entrevistada “C”, que já teve filhos, relatou sobre mudanças posturais que ocorreram durante a sua gravidez e os eventuais desconfortos que estas acarretaram. A partir disso, soube como lidar com tais situações, experimentando diversos exercícios que amenizavam os desconfortos, o que enriqueceu muito suas aulas para gestantes.

Essa questão tem a ver com a afirmação de Valadares (2002), que considera a gestante com o centro de gravidade deslocado à frente devido ao peso adquirido. Nesse sentido, há uma projeção do 
corpo para trás (lordose), um distanciamento dos pés, além da projeção à frente da cervical e tais alterações podem levar a lombalgia e cervicalgia.

Souza (2002) e Stephenson e O’Connor (2004) afirmam que os exercícios físicos praticados durante a gestação atenuam tais alterações, seja pelo aumento do tônus muscular, ou por evitar o aumento excessivo de peso.

A entrevistada "C" aponta outras diversas situações em que só teve clareza de como abordar a partir da sua própria gestação, como por exemplo, exercícios que na teoria são eficazes e na prática são inviáveis e vice-versa.

A entrevistada " $D$ " também afirma que a gravidez completou a sua visão teórica, no que diz respeito às alterações gravídicas e que através da experiência pessoal aprendeu a discernir sobre o que é aplicável realmente durante as atividades e o que não é, sempre, claro, respeitando a individualidade e a capacidade de suas alunas. Para ela:

Todos os profissionais que se dedicarem à sua área de trabalho, com estudo e 'experimentos' terão bom desempenho, mas no meu caso não houve melhor experiência do que vivenciar os nove meses de gestação, aplicando todo o conhecimento em mim mesma. Não foi tarefa fácil [...] agrega o sentimento de compartilhar toda realidade, lembrando que não se pode comparar uma mãe e outra, mas sim, estimular através de bons exemplos. (Entrevista realizada em 17 de agosto de 2012).

Nesta categoria, percebe-se que através das experiências apresentadas pelos professores no contato direto com a gestação e com o parto, essas situações tornaram-se elementos fundamentais para a competência na prestação de serviço no que se refere a planejar, executar e avaliar exercícios físicos para gestantes. A primeira informação relevante é que a maioria dos profissionais que participaram do estudo são mulheres e que muitas delas já passaram pela experiência de ter filhos. As próprias sensações e experimentos realizados durante a gravidez pelos professores apresentam um repertório de conhecimentos e de atividades que embasam sua prática. Até mesmo o participante do estudo do sexo masculino valorizou os momentos de acompanhamento da gestação e de parto de seu filho, o que ampliou o significado sobre essa fase de vida da mulher.

\section{A sensibilidade na pedagogia do ensino}

Inúmeros detalhes na periodização e no planejamento das atividades físicas devem ser levados em consideração para que os exercícios promovam as devidas melhorias na condição física e psicológica da 
gestante. Esta última diz respeito a melhor autoestima concebida principalmente nas aulas em grupo, em que há possibilidade de dividir experiências e anseios.

A forma de atuar dos profissionais está intimamente ligada ao seu perfil. Segundo a entrevistada “E”, gostar daquilo que se faz é imprescindível, principalmente quando se trata de públicos especiais.

Para a entrevistada "F":

Não é qualquer profissional que trabalha com gestantes, tem que ter feeling para saber até onde é uma reclamação de verdade, até onde é uma "manha". Se for este o caso, tem que saber lidar com essa situação, saber como passar as informações. (Entrevista realizada em 30 de agosto de 2012).

Ela complementa sua fala afirmando que é necessário ter feeling ${ }^{1}$ para trabalhar com as gestantes para entender até onde as reclamações delas são verdadeiras e até onde são manhas e diz que é necessário saber lidar com as duas situações.

Segundo o entrevistado "B":

Existem aulas em que é preciso ser mais paciente, em que elas vão te encher de perguntas sobre o que elas mesmas sentem. Em outros momentos vão querer exercitar-se "sem batepapo". Esses são exemplos simples do que precisamos estar preparados para lidar, sempre conciliando a situação em que elas se encontram no momento ao objetivo que as fazem estar ali praticando a atividade. (Entrevista realizada em 13 de agosto de 2012).

Além disso, o entrevistado "B" afirma que ter sensibilidade apurada durante as aulas implica na sua atuação e que em sua abordagem procura estar de acordo com o "astral" do momento.

Apesar dos participantes não especificarem se as aulas são personalizadas ou coletivas, Souza (2002) evidencia que em caso de insatisfação, baixa estima e melancolia, devido ao sobrepeso ou a questões emocionais, recomendam-se as atividades feitas em grupo que possibilitam a troca de experiências e a redução da ansiedade.

O entrevistado "B" relata que a sua forma de ministrar as aulas e de abordar os mais diversos assuntos que tangem a gestação envolvem todo o seu conhecimento técnico e específico e ele afirma que os exercícios físicos devem ser realizados com: “[...] muito amor em cada gesto”.

Percebe-se que as relações que vão sendo construídas entre o professor e as gestantes dão conta de valorizar o cuidado e a maneira pedagógica de expressão do profissional. Dessa forma, a sensibilidade, a maneira de ministrar a sessão de treinamento e a intimidade criada entre professor e aluna são

${ }^{1}$ Feeling: sensibilidade, sentimento ou pressentimento 
fundamentais para o sucesso do trabalho, dando mais segurança ao profissional e mais confiança à gestante.

\section{Conclusão}

De acordo com a análise das entrevistas que subsidiaram os resultados deste estudo, conclui-se que durante a graduação há poucos espaços para desenvolver conhecimentos sobre assuntos relacionados à mulher no período gestacional. Porém, é a partir da formação inicial que se constroem os conhecimentos básicos que são considerados aqueles ligados à área biológica do currículo.

Os conhecimentos específicos sobre as alterações fisiológicas que ocorrem na gravidez provêem na sua maioria de cursos de extensão. Porém, os sujeitos do estudo referenciam também a produção de estudos e uma formação específica oportunizada no local de trabalho. Os entrevistados julgam ser necessário o conhecimento profundo das alterações gravídicas devido ao fato de que somente tendo esta singularidade eles se sentem aptos a prescrever exercícios adequados à esta população em que haja um equilíbrio adequado de volume, intensidade, tempo de duração e resposta térmica.

Além das alterações fisiológicas, os entrevistados julgam ser necessários os conhecimentos de processos como o parto, a amamentação, as alterações posturais e os mais diversos assuntos que tangem à gestação e ao fato de "ser mãe". Esses temas aparecem como corriqueiros durante as aulas e é necessário passar certa segurança sobre eles durante as sessões. Para tal, ser mãe, pai, acompanhar gestações, acompanhar partos via internet ou pessoalmente são fatos que se mostraram enriquecedores ao conhecimento.

Surpreendentemente o entrevistado que ressaltou ser importante a sensibilidade e a afetividade para se trabalhar com gestantes foi o único homem participante do estudo. Considera-se que esses são quesitos importantes devido ao fato de que a mulher no período gestacional passa por diferentes emoções, medos e anseios e é preciso saber lidar com tais aspectos. Ser sensível a esse processo, analisar as informações palpáveis e emocionais e intervir de forma adequada é um fator importante para a maioria dos entrevistados.

Esses últimos quesitos estão intimamente ligados à forma de ministrar a aula, que cabe à pedagogia do ensino. O termo "pedagogia" não foi citado nas entrevistas, mas foi abordado através da forma como professor e aluno se relacionam durante as aulas. 
Entretanto, apesar do referencial teórico apontar para o fato de que durante a gestação as mulheres passam por uma revolução hormonal, nenhum dos entrevistados centrou seu discurso nesse aspecto, o que nos leva a pensar que conhecimentos de bioquímica também se apresentam como relevantes ao trabalho de intervenção proposto.

Dessa forma, conclui-se que às gestantes não é necessário restringir nenhum tipo de treinamento, desde que haja a liberação médica e maiores cuidados com mulheres sedentárias. Salienta-se, ainda, que os conhecimentos gravídicos são necessários, mas que uma pedagogia com sensibilidade também torna o trabalho de intervenção com as mulheres um processo mais competente.

\section{Referências}

ALMEIDA L. A. L.; CANDIDO, T. S.; OLIVEIRA NETTO, A. Conhecimento sobre a incontinência urinária e fisioterapia em gestantes: revisão de literatura. Revista Intersaúde. Jaú: V. 1, n. 2, p. 48-60, 2020.

BARDIN, L. Análise de conteúdo. 3 ed. Lisboa: Edições 70, 2004.

BATISTA, D. C. Atividade física e gestação: saúde dagestante não atleta e crescimento fetal. Rev. Bras. Saúde Matern. Infant., Recife, 3 (2): 151-158, abr. / jun., 2003.

CRESWELL, J. W. Projeto de pesquisa: método qualitativo, quantitativo e misto. 3 ed. Porto Alegre: Artmed, 2010.

EVANS, R. Mudanças fisiológicas. In: ORSHAN, S. A. Enfermagem na Saúde das Mulheres, das Mães e dos Recém-Nascidos: cuidado ao longo da vida. Porto Alegre: Artmed, 2010. p. 467-514.

GALLUP E. ET AL. Aspectos legais da prescrição de exercícios para agravidez. Seção III: Aplicações práticas. In: Artal R,Wiswell AR, Drinkwater LR. O exercício na gravidez.São Paulo: Manole; 1999. p. 293-8.

MENESTRINA, E. Educação Física e Saúde. 3. ed. Ijuí: Editora Unijuí, 2005.

MINAYO, M. C. S. O Desafio do Conhecimento: pesquisa qualitativa em saúde. 12 ed. São Paulo: Hucitec, 2010.

MOTTOLA, Michelle F. et al. 2019 Canadian guideline for physical activity throughout pregnancy. British Journal of Sports Medicine, v. 52, n. 21, p. 1339-1346, 2018.

PRATES, C. S.; SANTOS, L. C.; MORETTO, V. L. Modificações do organismo materno. In: OLIVEIRA, D. L. Enfermagem na Gravidez, Parto e Puerpério: notas de aula. Porto Alegre: UFRGS, 2005. p. 53-60. 
RAMOS, A. T. Atividade Física: diabetes, gestantes, $3^{\text {a }}$ idade, crianças, obesos. 3. ed. Rio de Janeiro: Sprint, 2002. p. 49-64.

SCHERER, A. et al. Diretrizes, propostas, interpretações e interesses - as dificuldades de diálogo na formação e na intervenção em educação física no Brasil. In: CAUDURO, M.T.; COCCO, F.; CARVALHO, N.O. (Org). A educação física nos seus diversos contextos: ensino - pesquisa - extensão. Curitiba: Editora CRV, 2017.

SOUZA. E. L. B. L. Exercício Físico na Gravidez. In: BARACHO, E. Fisioterapia Aplicada à Obstetrícia: aspectos em ginecologia e neonatologia. 3. ed. Rio de Janeiro: Médica e Científica LTDA, 2002. p. 129-140.

STEPHENSON, R. G.; O’CONNOR, L. J. Fisioterapia Aplicada à Ginecologia e Obstetrícia. 2 . ed. Barueri: Manole, 2004.

VALADARES, J. D. Adaptações fisiológicas da gestação. In SOUZA, E. L. B. L. Fisioterapia aplicada à obstetrícia: aspectos em ginecologia e neonatologia. Rio de Janeiro: Médica e Científica LTDA, 2002. cap. 2, p. 19-32.

ZAVORSKY G. S.; LONGO L. D. Exercise guidelines in pregnancy: new perspectives. Sports Med.New York: 41(5):345-60, 2011.

\section{Como citar este artigo}

SCHERER, A.; OLIVEIRA, M. S. M. P.; SCHERER, F. M. A atividade física para gestantes: análise da formação específica. Revista Kinesis, Santa Maria, v.38, p.01-16, 2020.

* O presente trabalho não contou com apoio financeiro de nenhuma natureza para sua realização. 